\title{
Auroral bremsstrahlung spectra in the atmosphere
}

\author{
J. G. LuhmanN \\ Space Sciences Laboratory, The Aerospace Corporation, El Segundo, California 90045, U.S.A.
}

(Received 12 July 1976; in revised form 4 October 1976)

\begin{abstract}
A procedure for computing the bremsstrahlung X-ray flux generated by auroral electrons in a plane, parallel atmosphere is described in detail. An integral expression which gives the $X$-ray flux at a specified photon energy and atmospheric depth for an arbitrary incident electron spectrum is set up. Some examples of $\mathrm{X}$-ray spectra obtained from the numerical solution of this multidimensional integral are illustrated and in several cases are compared with previous Monte Carlo results. The energy deposition by bremsstrahlung $\mathrm{X}$-rays can be determined from their (local flux spectra.
\end{abstract}

\section{INTRODUCTION}

The observation of $\mathrm{X}$-rays from within and above the atmosphere can provide global information about the location and intensity of kilovolt electron precipitation (e.g. Ghielmett et al., 1964, WILson et al., 1969). On the other hand, these X-rays can also provide an undesired background in the observation of extraterrestrial X-ray sources at balloon altitudes or at satellite altitudes when the viewing direction is near the horizon (HoLt, 1974). In either case, the relationship between the electron spectrum and the photon spectrum produced by the particles is an advantageous if not requisite area of information for interpreting the data. REES (1964), Kamiyama (1966) and Berger and SeltZER (1972) have employed various methods to compute the $\mathrm{X}$-ray flux spectrum that is generated in a plane, parallel atmosphere by an electron influx with a specific incident energy spectrum. These calculations are reviewed in a recent monograph by JONEs (1974). The procedure described in this paper, which produces results similar to those from the rigorous Monte Carlo calculations of Berger and Seltzer (1972), requires only the numerical evaluation of multidimensional integrals. Although Compton scattering is treated as an absorptive process and secondary electrons are neglected, the approximate method presented here provides an economical computational alternative for many practical applications. The economy with which these calculations can be performed is restricted primarily by the efficiency of the numerical integration routine employed and by the accuracy desired.

A crucial facet in the calculation of bremsstrahlung fluxes in the atmosphere is the description of the energy spectrum of the electrons which generate the $\mathrm{X}$-rays. Because electrons lose energy in matter by radiation and ionization processes, the electron spectrum changes as the particles penetrate into the air. In a previous report (LuHManN, 1976), an expression that describes the auroral electron spectrum at arbitrary altitude was derived for the case of an isotropic incident flux. Here this result is applied to the formulation of an integral expression that describes the electron initiated $X$ ray flux in a plane parallel atmosphere.

Only 'primary' X-rays resulting directly from the bremsstrahlung process are considered in the following derivation. Energy degraded 'secondary' photons produced by the Compton scattering of the bremsstrahlung photons are neglected. This omission, which greatly simplifies the calculation, restricts the practical application of the present method to predicting $\mathrm{X}$-ray intensities from electron spectra with the majority of flux below $\sim 40 \mathrm{keV}$. In most cases, auroral electron spectra satisfy this criterion (JoNEs, 1974). To demonstrate the accuracy of the approximate method described here, some X-ray spectra obtained by evaluating the flux integral are compared with the corresponding results from a Monte Carlo calculation (Berger and Seltzer, 1972). Several other examples of bremsstrahlung spectra are also calculated for various forms of incident electron spectra. Finally, the calculation of local energy deposition from the local photon spectrum is demonstrated.

\section{THEORY}

\section{(a) Electrons}

In a previous report (LuHManN, 1976) the spatial evolution of the energy spectrum of primary auroral electrons in the atmosphere during a stable, large scale influx was examined in detail. It was found that if pitch angle scattering and magnetic 
focussing are neglected, the Fokker-Planck equation describing the transport of auroral electrons in the atmosphere (WALt et al., 1969) can be solved analytically. More specifically, the Fokker-Planck equation

$$
\mu v \frac{\partial f}{\partial x}+\frac{\partial}{\partial T}\left[\frac{\mathrm{d} T}{\mathrm{~d} s} v f\right]=0
$$

where $f$ is the electron distribution function, $\mu$ is the cosine of the pitch angle, $x$ is the atmospheric depth in $\mathrm{g} \mathrm{cm}^{-2}, v$ and $T$ are particle velocity and kinetic energy respectively, and $\mathrm{d} T / \mathrm{d} s$ is the energy loss along the electron trajectory, has a solution given by

$$
f(\mu, x, T)=\frac{2 \pi}{v} \frac{\mathrm{d} T / \mathrm{d} s(\xi)}{\mathrm{d} T / \mathrm{d} s(T)} J_{0}(\xi)
$$

with

$$
\mathrm{d} T / \mathrm{d} s(Y)=\frac{Y^{-0.75}}{1.75 B}
$$

and

$$
\xi=\left\{\frac{1}{B} \frac{x}{\mu}+B T^{1.75}\right\}^{1 / 1.75}
$$

Here, $Y$ is a dummy variable, and $B$ is equal to $4.57 \times 10^{-6}$. The function $J_{0}$ describes the form of the incident electron spectrum $J_{0}(T)$. The definitions (3) and (4) originate from the empirical 'practical range' relation obtained by GRÜN (1957). For incident electrons isotropic over the downward hemisphere, the hemispherical electron flux spectrum $I$ at any atmospheric depth $x$ can then be described by the integral expression

$$
\begin{aligned}
I(x, T) & =\frac{v}{2 \pi} \int_{0}^{1} f \mathrm{~d} \mu \\
& =\int_{0}^{1} \frac{\mathrm{d} T / \mathrm{d} s(\xi)}{\mathrm{d} T / \mathrm{d} s(T)} J_{0}(\xi) \mathrm{d} \mu
\end{aligned}
$$$$
\text { electrons } / \mathrm{cm}^{2} \mathrm{keV}
$$

Details regarding the derivation of equation (5) are given in the original report.

\section{(b) Bremsstrahlung $X$-rays}

The bremsstrahlung $X$-ray production rate by a nonrelativistic electron of energy $T$ is described by the Bethe-Heitler differential cross section $\mathrm{d} \sigma_{\mathrm{B}} / \mathrm{d} k$ (Hertier, 1954). For an air target, the cross section presented by one gram of atoms is given approximately by

$$
\begin{aligned}
\frac{1}{M} \frac{\mathrm{d} \sigma_{B}}{\mathrm{~d} k}(k, T) & \\
& \cong \frac{1.75}{T k} \ln \left[\frac{(\sqrt{T}+\sqrt{T-k})^{2}}{k}\right] \mathrm{cm}^{2} / \mathrm{g} \mathrm{keV}
\end{aligned}
$$

where $M$ is the mean weight, in grams, of one atom of the atmospheric gas and $k$ is the photon energy in $\mathrm{keV}$ which has a maximum value equal to the electron energy $T$. The small variation of $M$ with altitude is neglected. The angular distribution of bremsstrahlung emission is incorporated by assuming that each photon is distributed in angle $\theta$ with respect to the direction of the electron that produced it according to the function (REES, 1964)

$$
\omega(\theta, T)=A(T) \frac{\sin ^{2} \theta}{(1-\beta \cos \theta)^{4}}
$$

where $A$, given by

$$
A(T)=2\left(1-\beta^{2}\right)^{5 / 2} / \pi
$$

is a normalization factor and $\beta=v / c$ is the electron speed divided by the speed of light.

The volume emissivity for bremsstrahlung $\mathrm{X}$ rays, which gives the production rate at depth $x$, energy $k$, and angle $\eta$ from the nadir, provided by the local electron population described by equation (2) can be obtained by integration:

$$
\begin{aligned}
P_{B}(x, k, \eta)= & \frac{1}{M} \int_{k}^{\infty} \mathrm{d} T\left\{\frac{\mathrm{d} \sigma_{B}}{\mathrm{~d} k} \int_{0}^{1} \mathrm{~d} \mu\right. \\
& \left.\times\left[\frac{\mathrm{d} T / \mathrm{d} s(\xi)}{\mathrm{d} T / \mathrm{d} s(T)} J_{0}(\xi) \int_{0}^{2 \pi} \omega(\theta, T) \mathrm{d} \tau\right]\right\}
\end{aligned}
$$

The units of $P_{B}$ as expressed above is photons $/(\mathrm{g} \mathrm{sec} \mathrm{keV})$. Here $\tau$ is the azimuthal angle of the photon direction measured from the vertical plane containing the electron vector. The geometry of photon emission is illustrated in Fig. 1. The angles $\theta, \tau$ and $\eta$ are related to the electron pitch angle cosine $\mu$ by the expression

$$
\cos \theta=\mu \cos \eta+\sqrt{1-\mu^{2}} \sin \eta \cos \tau
$$

The total flux of $X$-rays at a depth $X$ is a combination of both locally produced photons and contributions from the atmosphere above and below the altitude specified by $X$. The latter are attenuated by absorption in the atmosphere. For $\mathrm{X}$-rays in air, photoelectric absorption dominates below about $20 \mathrm{keV}$. Compton effects, which cause effective absorption at a given initial energy by changing the energy of the photon, become important at higher $X$-ray energies. In the following treatment, photons that undergo a Compton collision are assumed lost. Absorption itself, although caused by discrete interactions, will be regarded as a continuous process. Figure 2 shows the measured photon attenuation coefficients for oxygen and nitrogen as given by HubBell (1971). An average 


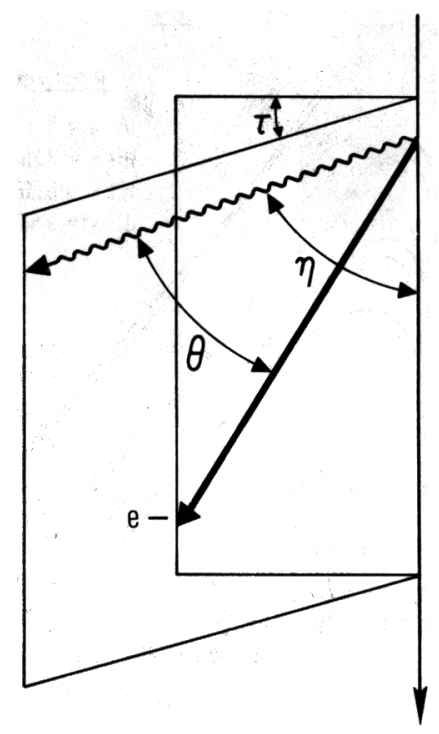

Fig. 1. Geometry of photon emission showing the orientations of the angles $\theta, \tau$ and $\eta$ with respect to the vertical

(x) axis and to the electron velocity vector $\left(e^{-}\right)$.

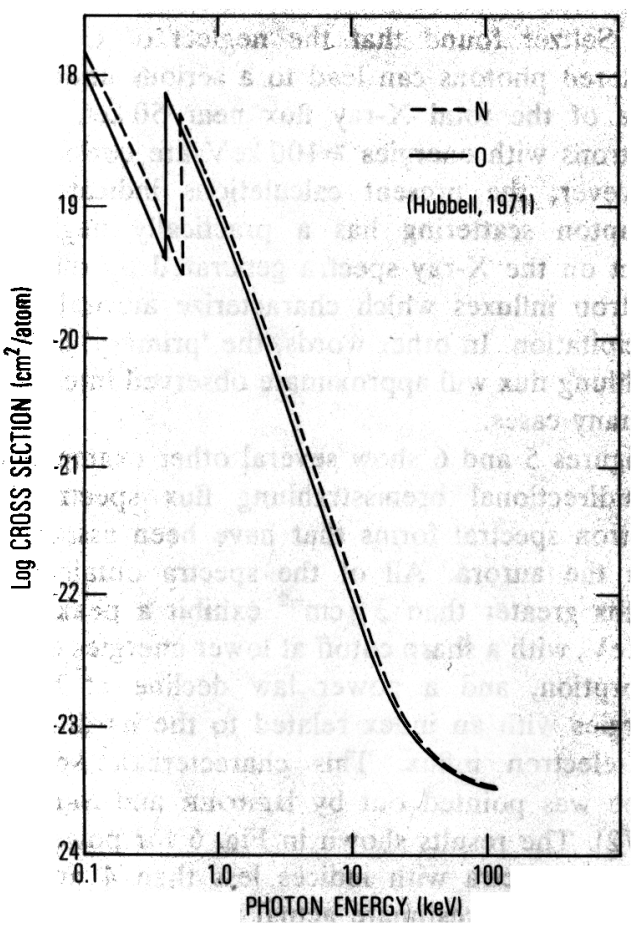

Fig. 2. Summary of photon absorption cross section measurements in oxygen and nitrogen after HUBBELL (1971). absorption cross section for air was obtained by weighting these values by the fractional contributions of oxygen and nitrogen to the atmosphere according to the CIRA 1965 model. Division of this average atomic cross section by the mean atomic weight $M$ gives the mass absorption coefficient $\mu_{a}(k)$ in $\mathrm{cm}^{2} / \mathrm{g}$.

The spatial evolution of the intensity of radiation $\mathscr{I}(x, k, \eta)$ in a medium where both photon production and absorption occur at energy $k$ is described by the equation of radiative transfer (CHANDRASEKHAR, 1950):

$$
\frac{\mathrm{d} \mathscr{I}}{\mathrm{d} x \sec \eta}=-\mu_{a} \mathscr{I}+\boldsymbol{P}
$$

Here, $\mathscr{I}$ has units of photons $/\left(\mathrm{cm}^{2} \mathrm{sec} \mathrm{keV}\right)$ and $P$ $(x, k, \eta)$ is the volume emissivity. For bremsstrahlung, $P$ is equal to $P_{B}$ (equation 9). The solutions to this transfer equation are well known. Upward or downward directional bremsstrahlung intensities viewed at depth $X$ are given by the source function $P_{B} / \mu_{a}$ multiplied by an exponential mass attenuation factor and integrated along the line of sight from the bottom or the top of the atmosphere to $X$ :

$$
\begin{aligned}
\mathscr{I}_{\text {up }} & =\int_{0}^{(1030-X) \sec \eta} S \mu_{a} \mathrm{~d} x \sec \eta \\
\mathscr{I}_{\text {down }} & =\int_{0}^{X \sec \eta} S \mu_{a} \mathrm{~d} x \sec \eta
\end{aligned}
$$

where

$$
S=\frac{P_{B}}{\mu_{a}} \exp \left[-\mu_{a}|X-x| \sec \eta\right]
$$

In the integrals (12) the depth $x=1030 \mathrm{~g} / \mathrm{cm}^{2}$ has been adopted as the base of the atmosphere. The $X$-ray fluxes through two hemispherical detectors bisected by the plane $x=X$ can then be obtained by integrating over the appropriate values of the photon direction angle $\eta$ :

$$
\begin{gathered}
\Phi_{\text {up }}(x, k)=\int_{\pi / 2}^{\pi} \Phi_{\text {up }} \mathrm{d} \eta \\
\Phi_{\text {down }}(x, k)=\int_{0}^{\pi / 2} \Phi_{\text {down }} \mathrm{d} \eta
\end{gathered}
$$

\section{APPLiCATIONS}

\section{(a) Local $X$-ray spectra}

The bremsstrahlung fluxes $\Phi_{\text {up }}$ and $\Phi_{\text {down }}$ as defined by equation (14) can now be calculated for 
any form of incident electron spectrum. Figures 3 and 4 show the omnidirectional flux spectra of unscattered bremsstrahlung $X$-rays at several atmospheric depths for the exponential incident electron spectrum

$$
J_{0}(T)=\frac{1}{\sim} \exp [-T / \alpha]
$$

with $\alpha$ equal to $10 \mathrm{keV}$ and $30 \mathrm{keV}$. The normalization factor $1 / \alpha$ gives results pertaining to unit incident electron flux. The spectrum for $x$ near zero, which describes the flux at the top of the atmosphere, has a negligible contribution from $\Phi_{\text {down }}$. In contrast, the spectra which characterize the deep atmosphere have negligible contributions from $\Phi_{\text {up. }}$. It was necessary to terminate the $\eta$ integration (equation 14) for $x \approx 0$ approximately 1 degree from the $\pi / 2$ limit because the assumption of an infinite plane atmosphere produces an unphysical divergence there.

The favorable comparison between the present calculation and the results of Berger and SELTZer (1972) is demonstrated by Figs. 3 and 4. Berger

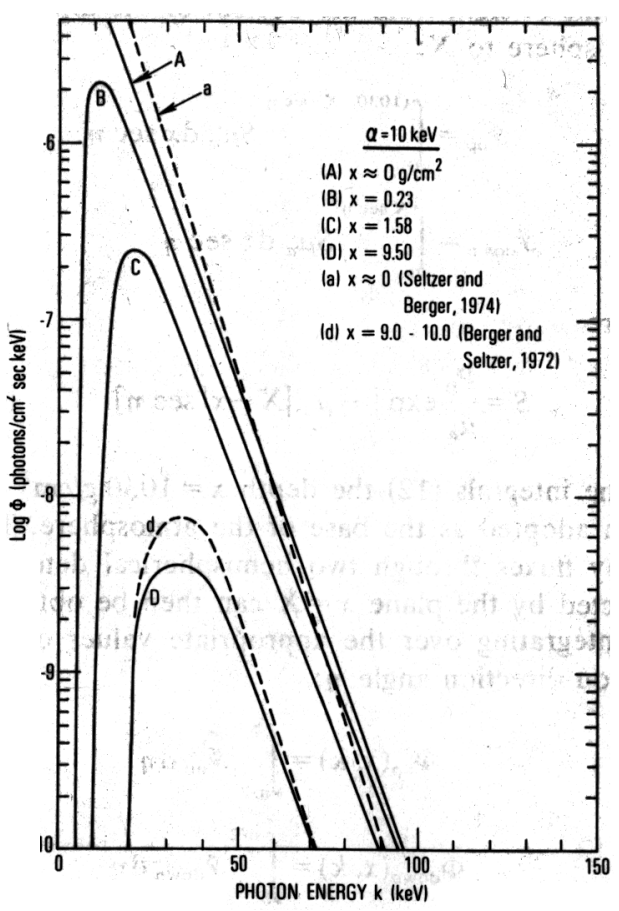

Fig. 3. Omnidirectional flux spectra of bremsstrahlung photons at several atmospheric depths for an incident exponential electron spectrum with an $e$-folding energy of $10 \mathrm{keV}$. The dashed lines show the results of previous Monte Carlo calculations (SELTZER and BERGER, 1974; Berger and Seltzer, 1972).

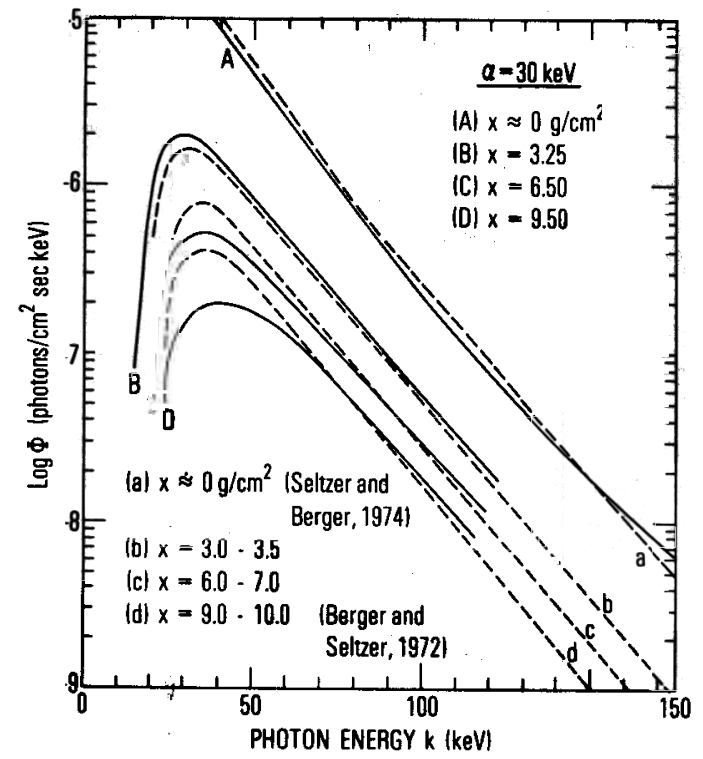

Fig. 4. Omnidirectional flux spectra of bremsstrahlung photons at several atmospheric depths for an incident exponential electron spectrum with an $e$-folding energy of $30 \mathrm{keV}$. The dashed lines show the results of previous Monte Carlo calculations (SELTZER and BERGER, 1974; Berger and Seltzer, 1972).

and Seltzer found that the neglect of Compton scattered photons can lead to a serious underestimate of the total X-ray flux near $50 \mathrm{keV}$ when electrons with energies $\geqslant 100 \mathrm{keV}$ are considered. However, the present calculations indicate that Compton scattering has a practically negligible affect on the X-ray spectra generated by the soft electron influxes which characterize auroral zone precipitation. In other words, the 'primary' bremsstrahlung flux will approximate observed intensities in many cases.

Figures 5 and 6 show several other examples of omnidirectional bremsstrahlung flux spectra for electron spectral forms that have been associated with the aurora. All of the spectra obtained at depths greater than $3 \mathrm{~g} \mathrm{~cm}^{-2}$ exhibit a peak near $40 \mathrm{keV}$, with a sharp cutoff at lower energies due to absorption, and a power law decline at higher energies with an index related to the hardness of the electron influx. This characteristic spectral shape was pointed out by BERGER and SELTZER (1972). The results shown in Fig. 6 for power law electron spectra with indices less than 4 are expected to underestimate actual flux levels because Compton scattered photons were neglected. Near $50 \mathrm{keV}$, the calculated flux may be as much as an order of magnitude deficient. However, the X-ray 


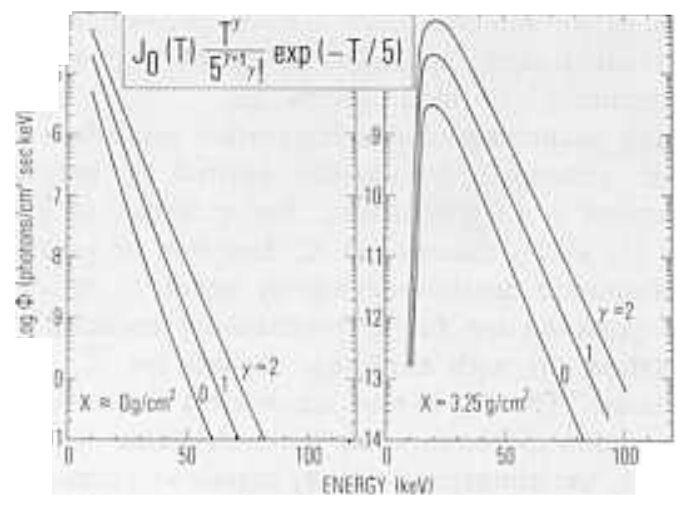

Fig. 5. Omnidirectional flux spectra of bremsstrahlung photons at the top of the atmosphere and at $3.25 \mathrm{~g} \mathrm{~cm}^{-2}$ for incident electron spectra with power law-exponential forms.

fluxes predicted in Figs. 3-5 for the soft electron auroras with power law exponential and exponential spectra are probably good approximations to the observable values.

\section{(b) Energy deposition profiles}

In a previous report (LummanN, 1976) the altitude profiles of the energy deposited in the atmosphere by electron impact ionization were discussed. The $\mathrm{X}$-rays that are generated by electron precipitation also deposit energy in the atmosphere through photoelectric and Compton absorption. Because they are on the average more penetrating than the electrons, the auroral $X$-rays, which represent only a small fraction of the total energy in the incident electron stream, can dominate the energy deposition in the lower $D$-region of the ionosphere.

$\mathrm{X}$-ray energy deposition vs altitude profiles can

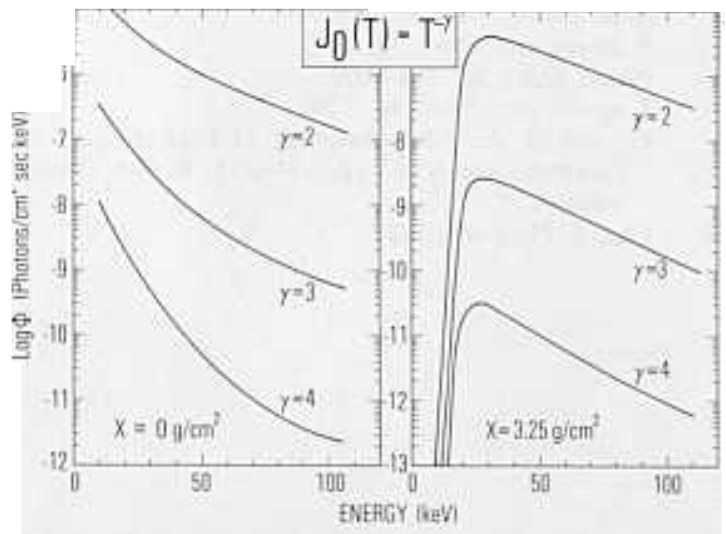

Fig. 6. Omnidirectional flux spectra of bremsstrahlung hhotons at the top of the atmosphere and at $3.25 \mathrm{~g} \mathrm{~cm}^{-2}$

for incident electron spectra with power law forms. be obtained from the X-ray flux if it is assumed that the photon energy is deposited at the site of its absorption. This assumption is justified whenever the photoelectric effect provides the dominant absorption mechanism because photoelectrons generally have such low energies following their ejection that they travel only a short distance from their point of origin. The energy deposited by $\mathrm{X}$-rays in the atmosphere is described by the integral (Berger et al., 1974)

$$
A_{u}(x)=\int_{n}^{\infty} \mu_{u}\left[\Phi_{u p}(x, k)+\Phi_{\text {down }}(x, k)\right] k d k
$$

where the flux $\Phi(x, k)$ can be obtained from equation (14). The energy deposition function $A_{B}(x)$ has units of $\mathrm{keV} / \mathrm{cm}^{2} \sec \left(\mathrm{g} \mathrm{cm}^{-2}\right)$. Deposition in $\mathrm{keV} / \mathrm{cm}^{3} \mathrm{sec}$ is obtained by multiplying $A_{B}$ by the mass density $\rho(x)$ in $\mathrm{g} / \mathrm{cm}^{3}$. Here the density function $\rho(x)$ from the CIRA 1965 Mean Atmosphere will be assumed for comparison with the results of Berger et al.

The bremsstrahlung deposition function $A_{B}$ at three altitudes was evaluated for an incident exponential electron spectrum with $\alpha$ equal to $10 \mathrm{keV}$. The $\mathrm{X}$-ray spectra in Fig. 3 give $A_{B}$ at approximately 60,45 and $32 \mathrm{~km}$, which correspond to $0.23,1.58$ and $9.50 \mathrm{~g} \mathrm{~cm}^{-2}$ respectively if the CIRA 1965 atmosphere is invoked. These points are plotted in Fig. 7. To obtain more detailed altitude coverage, many local X-ray spectra could be constructed. The deposition profile from impact ionization (LuHManN, 1976) is shown for comparison

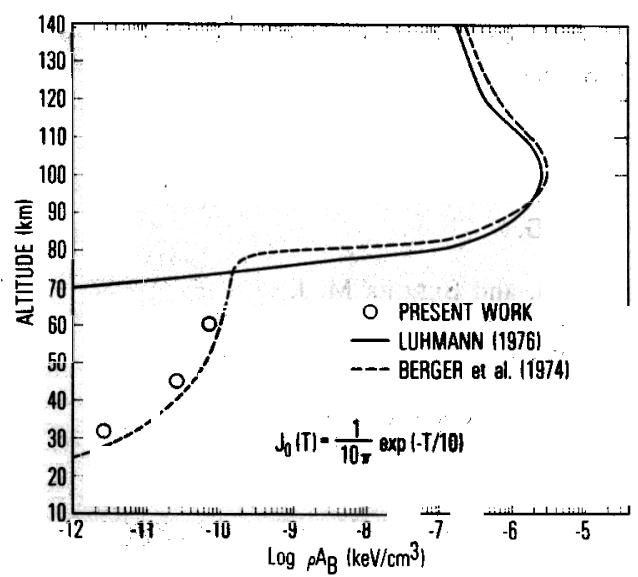

Fig. 7. Energy deposition profile for an incident exponential electron spectrum with an $e$-folding energy of $10 \mathrm{keV}$. The peak at $100 \mathrm{~km}$ is the result of impact ionization. Below $70 \mathrm{~km}$ the energy is deposited by bremsstrahlung $\mathrm{X}$-rays. These results are normalized to unit incident electron current. The dashed line is derived from a previous Monte Carlo calculation (BERGER et al., 1974). 
together with a total energy deposition profile derived from the results of BERGER et al. (1974). These profiles emphasize the need for including auroral bremsstrahlung as an energy source for the lower ionosphere.

\section{CONCLUDING REMARKS}

A method for computing the bremsstrahlung $\mathrm{X}$ ray fluxes generated by auroral electrons in an infinite plane, parallel atmosphere has been described in detail. The agreement between the results of these calculations, and the results of Berger and Seltzer (1972) demonstrates that this procedure can provide a useful alternative to the Monte Carlo approach. Like the Monte Carlo calculation, this numerical integration method allows the prediction of the upward and downward flux spectra of bremsstrahlung X-rays at arbitrary altitude. It provides a particularly efficient means of finding the auroral $X$-ray flux at a few specific d.sth and energy points in cases where the assump- tion of an infinite, plane parallel atmosphere is justified. Energy deposition by the X-rays can be determined from their spectra.

The adaptation of this integration procedure to other atmospheric emissions excited by auroral electrons is straightforward. For example, to find the $\mathrm{O}_{2}$ or $\mathrm{N}_{2}$ fluorescent $K_{\alpha}$ line flux at various atmospheric depths one merely needs to replace the cross section for bremsstrahlung emission in equation (9) with the cross section for $K_{\alpha}$ line emission. The $K_{\alpha} \mathrm{X}$-rays are subject to the same absorption mechanisms as bremsstrahlung $\mathrm{X}$-rays. Hence, the atmospheric $X$-ray emissions excited by various forms of auroral electron spectra can be predicted rather easily once a code is set up to perform the bremsstrahlung calculation.

Acknowledgement-This work was conducted under U.S. Air Force Space and Missile Systems Organization (SAMSO) Contract No. F04701-75-C-0076. The author would like to thank Dr. J. B. BLAKE for his comments and advice during the course of this work.
Berger M. J. and Seltzer S. M.

Berger M. J., Seltzer S. M. and MAEdA K.

Chandrasekhar S.

CIRA

Ghielmetti H. S., Becerra N., Godel A. M., Heredia H. and ROEDERER G.

GRüN A. E.

HeItLER W.

Holt S. S. (Ed.

Hubbell J. $\mathbf{H}$.

JONES A. V.

KamiYama $\mathrm{H}$.

LUHMANN J. G.

REES M. H.

Seltzer S. M. and Berger M. J.

WALT M., MCDONALD W. M. and Francis W. E.

Wilson B. G., BAXter A. J. and GreEN D. W.

\section{REFERENCES}

$1972 \quad J$. atmos. terr. Phys. 34, 85.

1974 J. atmos. terr. Phys. 36, 591.

1950 Radiative Transfer. Clarendon Press, Oxford.

1965 COSPAR International Reference Atmosphere. North Holland, Amsterdam.

1964 Phys. Rev. Lett. 12, 388.

1957 Z. Naturf. 12a, 89.

1954 The Quantum Theory of Radiation. Clarendon Press, Oxford.

1974 Proc. Workshop on Electron Contamination in X-ray Astronomy Experiments. NASA X-661-74-130.

1971 Atomic Data 3, 241.

1974 Aurora. Reidel, Dordtrecht.

1966 Rept. Ionos. Space Res. 20, 374.

$1976 J$ J. atmos. terr. Phys. 38, 605.

1964 Planet Space Sci. 12, 1093.

1974 J. atmos. terr. Phys. 36, 1283.

1969 Physics of the Magnetosphere (Edited by R. L. Carovillano et al.) pp. 534-555. Reidel, Dordtrecht.

1969 Can. J. Phys. 47, 2427. 